\title{
The direct superior approach versus posterior approach for total hip arthroplasty: study protocol for a prospective double-blinded randomised control trial
}

\author{
Babar Kayani* (D), Sujith Konan, Jenni Tahmassebi, Atif Ayuob and Fares S. Haddad
}

\begin{abstract}
Background: The direct superior approach (DSA) is a minimally invasive modification of the posterior approach $(\mathrm{PA})$ that preserves the iliotibial band and short external rotators except for the piriformis or conjoint tendon during total hip arthroplasty (THA). The objective of this study is to compare patient satisfaction, functional outcomes, accuracy of implant positioning, component stability, gait, cost-effectiveness, and complications in the DSA versus PA for THA.

Methods and analysis: This prospective double-blinded randomised control trial will include 80 patients with symptomatic hip osteoarthritis undergoing primary THA. Following informed consent, patients will be randomised to THA using the PA (control group) or DSA (investigation group) at a ratio of 1:1 using an online random number generator. Blinded observers will review patients at regular intervals for 2 years after surgery to record predefined study outcomes relating to postoperative rehabilitation, clinical progress, functional outcomes, accuracy of implant positioning, gait analysis on force plate treadmill, implant migration with radiosteriometric analysis, costeffectiveness, and complications. A superiority study design will be used to evaluate whether the DSA provides improved outcomes compared to the PA for THA. Evaluation of study outcomes in DSA and PA will be used to quantify and draw inferences on differences in the efficacy of treatment between the two groups. Intention-to-treat and per-protocol population analysis will be undertaken. The following statistical methods will be employed to analyse the data: descriptive statistics, independent $t$ test, paired $t$ test, analysis of variance, Fisher exact test, chisquare test, and graphical displays. Ethical approval was obtained from the London-Fulham Research Ethics Committee, UK. The study is sponsored by University College London, UK.
\end{abstract}

Discussion: This study compares a comprehensive and robust range of clinical, functional, and radiological outcomes in THA performed using the PA versus DSA. The findings of this study will provide an improved understanding of the differences in the PA versus DSA for THA with respect to patient satisfaction, functional outcomes, implant survivorship, gait, cost-effectiveness, and complications.

Trial registration: ClinicalTrials.gov, NCT04191993. Registered on 10 December 2019

\footnotetext{
* Correspondence: babar.kayani@gmail.com

Department of Trauma and Orthopaedic Surgery, University College Hospital,

235 Euston Road, Fitzrovia, London NW1 2BU, UK
}

(c) The Author(s). 2020 Open Access This article is licensed under a Creative Commons Attribution 4.0 International License, which permits use, sharing, adaptation, distribution and reproduction in any medium or format, as long as you give appropriate credit to the original author(s) and the source, provide a link to the Creative Commons licence, and indicate if changes were made. The images or other third party material in this article are included in the article's Creative Commons licence, unless indicated otherwise in a credit line to the material. If material is not included in the article's Creative Commons licence and your intended use is not permitted by statutory regulation or exceeds the permitted use, you will need to obtain permission directly from the copyright holder. To view a copy of this licence, visit http://creativecommons.org/licenses/by/4.0/ The Creative Commons Public Domain Dedication waiver (http://creativecommons.org/publicdomain/zero/1.0/) applies to the data made available in this article, unless otherwise stated in a credit line to the data. 


\section{Background}

Total hip arthroplasty (THA) is an effective procedure for relieving pain, restoring function, and improving the quality of life in patients with end-stage hip osteoarthritis $[2-5,7,16,20]$. Analysis of joint registry data from the UK, Sweden, and New Zealand has shown that the posterior approach (PA) is the most commonly used approach for THA [16]. The main advantages of the PA for THA are that it preserves the abductor mechanism, reduces intraoperative blood loss, and decreases the risk of heterotrophic ossification compared to the anterolateral and direct lateral approaches $[10,14,16]$. However, the PA is associated with increased risk of sciatic nerve injury during dissection, bleeding from the inferior gluteal artery as it leaves the pelvis below the piriformis, and increased risk of dislocation compared to these other approaches $[6,8,11,25]$. Evolution in minimally invasive surgery and recent innovations in surgical instrumentation have led to the development of the direct superior approach (DSA), which is a modification of the PA that preserves the iliotibial band and the short external rotators except for the piriformis or conjoint tendon $[17,19,21]$. Conceptually, improved preservation of this periarticular soft tissue envelope may help to reduce postoperative pain, improve functional recovery, and better restore native hip biomechanics $[13,18]$.

Existing studies on the DSA for THA are casecontrolled trials or case series with limited data on validated functional outcomes or clinically significant radiological outcomes [17, 19, 21]. Nam et al. conducted a prospective non-randomised trial comparing THA performed using 196 PAs versus 42 DSAs at three separate treatment centres [17]. The authors reported there was no difference in moderate to severe pain over the greater trochanter, anterior thigh, and lateral thigh between the two approaches at a minimum 1-year follow-up. Patients undergoing the PA had improved University of California at Los Angeles hip (UCLA) scores compared to those undergoing the DSA at 1-year follow-up. However, this study included different operating surgeons within three healthcare institutions, and the PA was performed through a modified mini-incision in patients that were younger and more active than the DSA group. Roger and Hill retrospectively reviewed outcomes in a case series of 135 patients undergoing the DSA for THA and reported good postoperative functional outcomes as assessed using the Harris hip score at 1-year follow-up [21]. Mean acetabular cup abduction was $41^{\circ}$ (range $21-$ $\left.49^{\circ}\right)$, and mean acetabular cup anteversion was $21^{\circ}$ (range $15-27^{\circ}$ ). Femoral implant positioning greater than $2^{\circ}$ varus and valgus was observed in $4 \%$ and $2 \%$ of patients respectively. This study was a retrospective study with no control group and heterogeneity in postoperative follow-up times.
Penenberg et al. followed 250 patients undergoing the DSA for THA and found mean Harris hip scores improved from 47.71 preoperatively to 95.6 within 3 to 6 months postoperatively [19]. Femoral implant positioning was within $2^{\circ}$ of the planned position in $97 \%$ of patients with mean acetabular cup abduction of $42^{\circ}$ (range 30 to $55^{\circ}$ ) and acetabular cup anteversion of $31^{\circ}$ (range 22 to $40^{\circ}$ ) in all study patients. This was a retrospective study with limited follow-up, no control group, patients and clinicians were not blinded, and statistical analysis for assessing the significance of study outcomes was not performed. Amanatullah et al. assessed periarticular muscle injury in eight cadaveric specimens in which the direct anterior approach was performed on one side and the DSA for THA on the contralateral side [1]. The DSA was associated with reduced iatrogenic injury to the gluteus minimus muscle, gluteus minimus tendon, tensor fascia lata, and rectus femoris compared to the direct anterior approach. This study had a small sample size and iatrogenic soft tissue injury was graded in cadaveric specimens, and therefore, it remains unknown how the observed differences in muscle injury translate to clinical outcomes between the two treatment groups.

There remains a paucity of high-quality evidence comparing clinical, functional, and radiological outcomes in the PA versus DSA for THA. Further, it remains unknown how differences in muscle preservation in the DSA and PA translate to postoperative pain scores between the two treatment groups. It is possible to improve on existing studies by assessing a more comprehensive and robust range of outcome measures, prospectively randomising patients to their respective treatment groups, standardising the surgical techniques within each group, using the same implant designs and postoperative rehabilitation protocol in all study patients, and blinding patients and observers recording study outcomes. Radiosteriometric analysis (RSA) will also be used to assess implant micromotion, which correlates with component loosening and provides prognostic information on long-term implant survivorship [2224 ]. The findings of this study will provide an improved understanding of the differences in the PA versus DSA for THA with respect to patient satisfaction, functional outcomes, accuracy of implant positioning, component survivorship, cost-effectiveness, and complications.

\section{Methods/design \\ Objectives}

The primary objective of this study is to compare postoperative pain scores on the visual analogue scale (VAS) in the PA versus DSA for THA. The study hypothesis is that the DSA will reduce periarticular soft tissue dissection that will translate to reduced postoperative pain scores on the VAS compared to the PA for THA. 
The secondary objectives are to compare the following outcomes between the two treatment groups:

1. Surgical efficiency

2. Postoperative functional rehabilitation

3. Patient satisfaction

4. Functional outcomes

5. Quality of life

6. Muscle group strength

7. Range of motion

8. Accuracy of restoring planned hip biomechanics

9. Accuracy of achieving planned component positioning

10. Implant stability

11. Gait analysis

12. Resource use and cost-effectiveness

13. Complications

\section{Trial design}

This study is a prospective, single-centre, doubleblinded, randomised control trial. The study will be undertaken in the Department of Trauma and Orthopaedics, University College Hospital, 235 Euston Road, Bloomsbury, London NW1 2BU, UK. The study will include 80 patients randomly allocated to either PA (control group) or DSA (investigation group) for THA. The study commenced patient recruitment in June 2018 and is expected to complete patient recruitment in December 2020. All patients will be followed up for 2 years after surgery, and therefore, the anticipated completion date for the study is December 2022. The study is sponsored by University College London, UK. The patient enrolment flowchart is presented in Fig. 1. The schedule of enrolment, interventions, and assessments for all study patients is shown in Fig. 2.

\section{Eligibility criteria}

The inclusion criteria for this study are as follows: the patient has symptomatic hip osteoarthritis requiring primary THA; the patient is fit for surgical intervention following review by a surgeon and anaesthetist; the is patient aged between 18 and 80 years at the time of surgery; the patient is able to give informed consent and agrees to comply with the postoperative review programme; and the patient has sufficient mobility to attend follow-up clinics. The exclusion criteria for this study are as follows: patient is undergoing revision surgery or second-stage THA; patients in whom the planned hip biomechanics are in a different position to the contralateral hip (e.g. developmental dysplasia of the hip or protrusio acetabuli); patient is not suitable to have the planned study implants (e.g. requiring dual mobility component or cemented implants); patient is immobile or has another neurological condition affecting musculoskeletal function; patient is already enrolled on another concurrent clinical trial; patient is unable or unwilling to sign the informed consent form specific to this study; and patient is unable to attend the study followup programme. Importantly, patients that do not receive the planned study implants (e.g. intraoperative assessment showing poor bone quality or bone loss necessitating cemented implants for THA) will be excluded from the study.

\section{Recruitment}

Patients will be recruited from the orthopaedic outpatient clinic at University College Hospital, London, UK. All patients will be screened by the clinical team (orthopaedic consultant surgeon, clinical research fellow, and orthopaedic registrar) for study participation based on the predefined inclusion and exclusion criteria listed above. Patients that fulfil the eligibility criteria and express an interest to participate in the study will be provided with an ethics committee-approved patient information sheet. This provides details about the study treatment, follow-up, and contact details for further information. All members of the clinical team are familiar with the study and will address any preliminary questions about the study. Details of those patients expressing an interest to participate in the study will be recorded in the patient contact form and forwarded to the research physiotherapist. The research physiotherapist will phone the patient 4 weeks after this consultation to discuss any further questions and confirm if the patient would like to participate in the study.

\section{Consent}

Informed consent will be obtained by the chief investigator or principal investigator when the patient attends for preoperative assessment. This is 6 weeks after the outpatient consultation for agreement to THA and 2 weeks before surgery. It is important to the data collection scheme that patients are able to follow commands, read, and interpret questions via questionnaires. For those who cannot hear, read, or understand English, an interpreter will be provided. Identical preoperative imaging modalities for surgical planning will be used in both treatment groups.

\section{Allocation}

After informed consent has been obtained, the research physiotherapist will randomise the patient into one of the two treatment groups using an online random number generator (www.random.org). A number from 1 to 80 can be randomly generated and will allocate a patient to one of the two arms of the study: 1-40 inclusive for the control group and 41-80 inclusive for the investigation group. The research physiotherapist will perform 


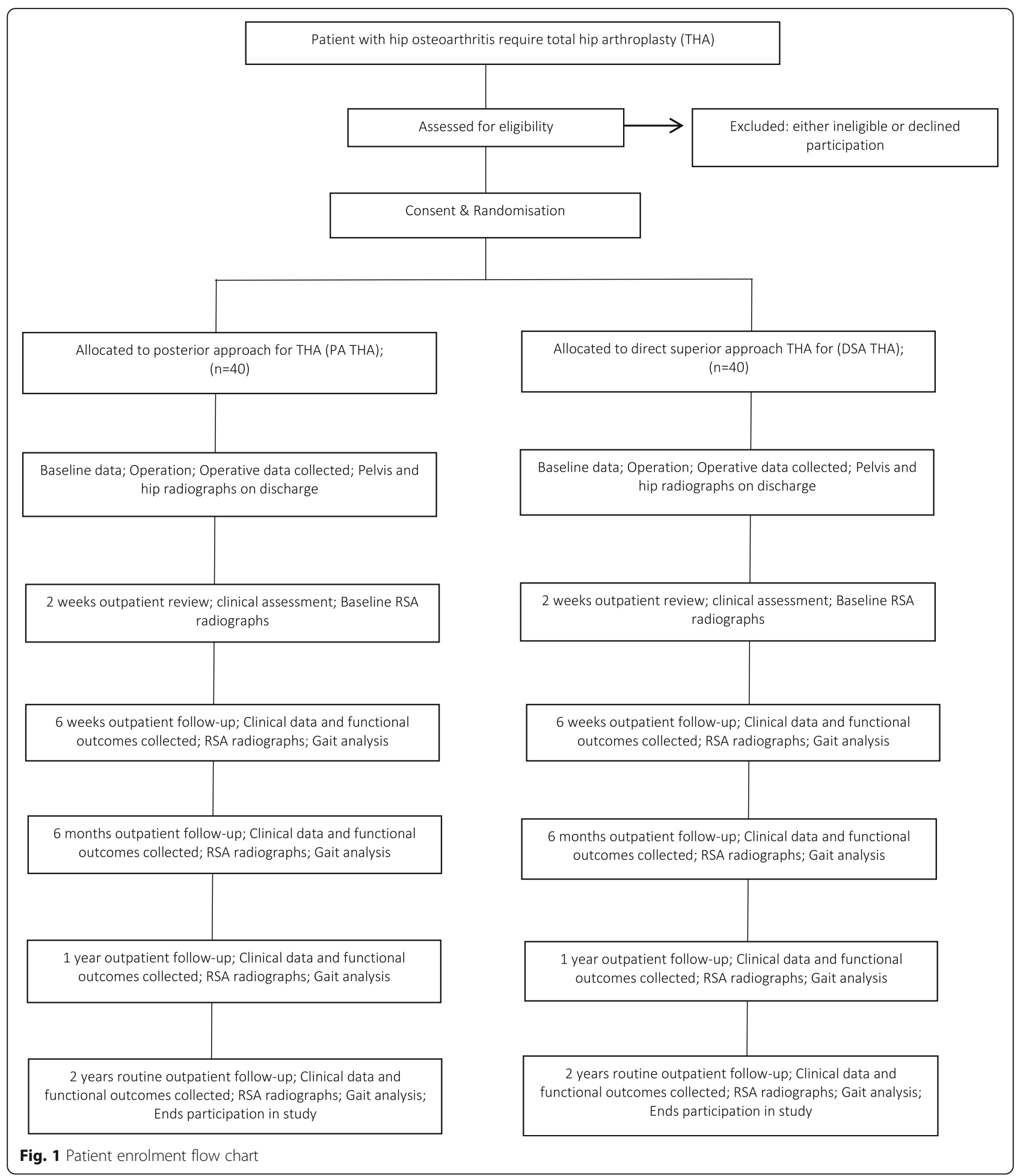

the randomisation procedure and store the designated treatment group for each patient on a passwordencrypted file on the hospital computer. The operating surgeon will have this information communicated to them on the morning of surgery.

\section{Preoperative imaging}

All patients will undergo preoperative imaging with plain pelvic and hip radiographs. In both treatment groups, pelvic radiographs will be exported onto Traumacad software (Traumacad, Petach-Tikva, Israel) to template 


\begin{tabular}{|c|c|c|c|c|c|c|c|c|}
\hline \multirow[b]{3}{*}{ TIMEPOINT } & \multirow{3}{*}{$\begin{array}{l}\text { Enrolment } \\
-8 \text { weeks }\end{array}$} & \multicolumn{7}{|c|}{ STUDY PERIOD } \\
\hline & & \multirow{2}{*}{$\begin{array}{c}\text { Pre-op CT } \\
-2 \text { weeks }\end{array}$} & \multirow{2}{*}{$\begin{array}{c}\text { Allocation } \\
0\end{array}$} & \multicolumn{4}{|c|}{ Post-allocation } & \multirow{2}{*}{$\begin{array}{c}\begin{array}{c}\text { Close- } \\
\text { out }\end{array} \\
2 \text { year }\end{array}$} \\
\hline & & & & 2 weeks & 6 weeks & 6 months & 1 year & \\
\hline \multicolumn{9}{|l|}{ ENROLMENT: } \\
\hline Eligibility screen & $x$ & & & & & & & \\
\hline Informed consent & & $x$ & & & & & & \\
\hline Allocation & & & $x$ & & & & & \\
\hline \multicolumn{9}{|l|}{ INTERVENTIONS: } \\
\hline PA THA & & & $x$ & & & & & \\
\hline DSA THA & & & $x$ & & & & & \\
\hline \multicolumn{9}{|l|}{ ASSESSMENTS: } \\
\hline $\begin{array}{r}\text { Demographics \& } \\
\text { Baseline variables }\end{array}$ & & & $\mathrm{x}$ & & & & & \\
\hline Operative outcomes & & & $x$ & & & & & \\
\hline $\begin{array}{r}\text { Pain and Analgesia } \\
\text { Requirements }\end{array}$ & & $x$ & & & $\mathrm{x}$ & $x$ & $x$ & $\mathrm{x}$ \\
\hline $\begin{array}{r}\text { Patient-reported } \\
\text { outcome measures }\end{array}$ & & $x$ & & & $x$ & $x$ & $x$ & $x$ \\
\hline Clinical examination & $x$ & & $x$ & & $x$ & $x$ & $x$ & $x$ \\
\hline \multirow{2}{*}{$\begin{array}{r}\text { Adverse events } \\
\text { Plain pelvis and hip } \\
\text { radiographs }\end{array}$} & $\mathrm{X}$ & $\mathrm{x}$ & $\mathrm{x}$ & $\mathrm{x}$ & $\mathrm{X}$ & $x$ & $\mathrm{X}$ & $x$ \\
\hline & $x$ & & $x$ & & & & & \\
\hline RSA Radiographs & & & & $x$ & $x$ & $x$ & $x$ & $x$ \\
\hline Gait analysis & & & & $\mathrm{x}$ & $x$ & $\mathrm{x}$ & $x$ & $x$ \\
\hline \multicolumn{9}{|c|}{$\begin{array}{l}\text { Legend: PA THA - Posterior approach total hip arthroplasty; DSA THA - Direct superior approach total hip } \\
\text { arthroplasty; RSA-Radiosteriometric analysis }\end{array}$} \\
\hline \multicolumn{9}{|c|}{$\begin{array}{l}\text { Fig. } 2 \text { Schedule of enrolment, interventions, and assessments for all study patients. PA THA posterior approach total hip arthroplasty, DSA THA } \\
\text { direct superior approach total hip arthroplasty, RSA radiosteriometric analysis }\end{array}$} \\
\hline
\end{tabular}

optimal implant positioning and sizes for achieving the planned bone coverage, component version and inclination, horizontal and vertical centres of rotation, acetabular and femoral offset, and leg-length correction. All preoperative templating will be undertaken by the operating surgeon 2 weeks prior to surgery. Preoperative pain scores on the VAS, analgesia requirements, and functional outcome scores (Fig. 2) will also be collected preoperatively as baseline values.

\section{Surgical intervention}

All operative procedures will be performed by one of two consultant orthopaedic surgeons that are fully trained and experienced with both the PA and DSA for 
THA. In both treatment groups, the patient will be placed in the lateral decubitus position and a curved incision performed behind the posterior edge of the greater trochanter extending distally towards the shaft of the femur. This initial incision will be $8-10 \mathrm{~cm}$ in length but can later be extended as required. The fascia lata will be incised along the length of the incision and the fibres of the gluteus maximus split along the line of the incision to expose the underlying pericapsular fat. Retractors will be used to retract the gluteus medius anteriorly and the soft tissue along the posterior border of the proximal femur inferiorly. The pericapsular fat will be removed with diathermy and the plane between the gluteus medius and gluteus minimus muscle developed. The hip joint will then be internally rotated to place the short external rotators on stretch. In the PA, diathermy will be used to detach the external rotators (including piriformis) close to their femoral insertion. In the DSA, caution will be taken to identify and protect the iliotibial band during dissection (Fig. 3) [12, 19, 21]. A retractor will be placed directly under the obturator internus to protect the inferior gemellus muscle below. The piriformis (or conjoint tendon) will be detached as close to their femoral insertions as possible but the remaining external rotators preserved. The capsulotomy will start at the distal, inferolateral aspect of the wound and extend proximally and posteromedially towards the superior acetabular margin. The capsule will be elevated subperiosteally to create superior and inferior capsular flaps. The retractors will then be repositioned inside the capsule and the hip dislocated posteriorly by flexion, adduction, and internal rotation.

The femoral osteotomy site will be marked using the templated measurements from the greater and lesser trochanters with the femoral neck cutting guide in place. An oscillating saw will be used to perform the osteotomy with Hohmann retractors protecting the surrounding soft tissues. The femoral osteotomy will be performed with the saw blade $45^{\circ}$ to the femoral shaft and in the plane of the tibia. Sharp Hohman retractors will be positioned over the anterior wall to lever the femur anteriorly and under the transverse acetabular ligament to expose the whole acetabulum for preparation. Soft tissues overhanging the acetabular circumference will be excised. Osteophytes will also be excised with an osteotome and the medial wall visualised. Hemispherical reamers will be used to remove the residual acetabular cartilage and expose the underlying subchondral bone. In the PA, this will be undertaken using sequentially larger straight reamers until the medial wall is reached. In the DSA, medial acetabular wall reaming will initially be undertaken using a straight acetabular reamer and then sequentially larger angled reamers until the medial wall is reached $[19,21]$. In both groups, an external alignment guide will be attached to the cutting-edge reamer handle and acetabular impactor to improve the accuracy of acetabular cup positioning. Acetabular cup positioning will be guided by the preoperative surgical plan and transverse acetabular ligament to match the patient's native acetabular cup inclination and version within Lewin-

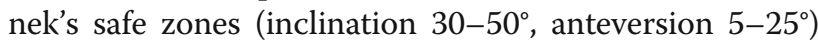
[15]. Any residual osteophytes will be removed at this stage using an osteotome and bone nibbler, and the trial prosthesis inserted to ensure satisfactory coverage and stability. Line-to-line technique for acetabular implantation will be used with implantation of the acetabular cup that is the same size as the last reamer used, and this will be augmented with two acetabular screws. The leg will then be placed into $40^{\circ}$ flexion, $40^{\circ}$ internal rotation, and $40^{\circ}$ abduction to visualise the femoral osteotomy site. A femoral elevator will be inserted under the anterior femoral neck to elevate and expose the proximal

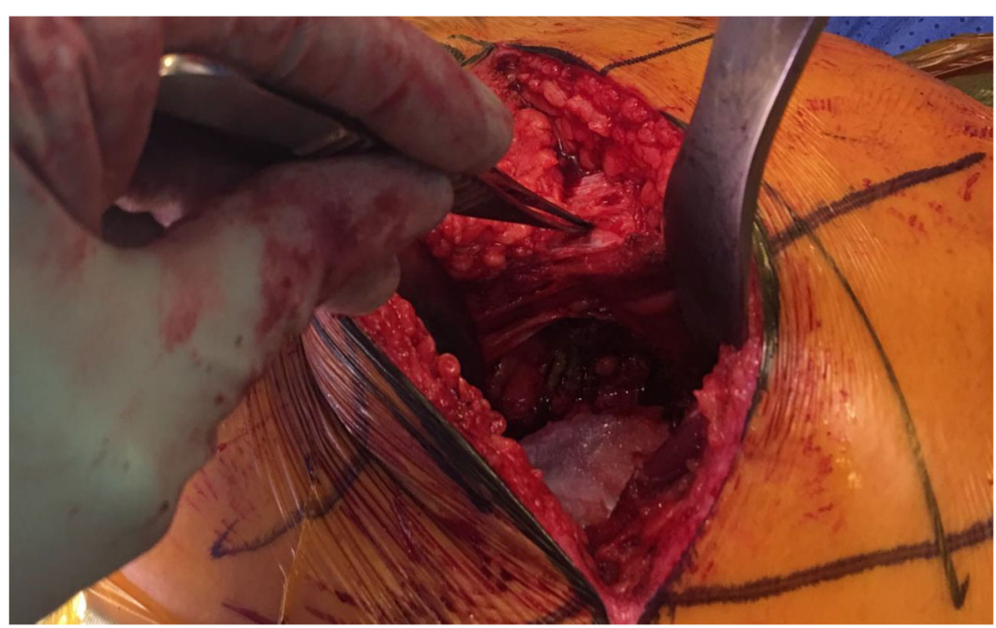

Fig. 3 intraoperative photo showing the preservation of the iliotibial band. 
femur, and a box chisel used to remove bone from the posterolateral femoral neck. The femur will be prepared using a rasp and sequential broaching until maximum cortical contact in the mediolateral dimension is achieved. The femur will be prepared and implanted with $10-20^{\circ}$ anteversion. Trial femoral heads will be applied to check hip joint stability, offset, soft tissue tension, and leg-length discrepancy prior to definitive selection of the femoral head. The final femoral stem will then be implanted, femoral head applied to the taper, and hip joint reduced. The capsule and external rotators will be repaired back to the femur and a layered closure of the fascia, subcutaneous tissue, and skin performed.

All study patients will receive the Accolade II femoral stem (Stryker Ltd, Mahwah, NJ, USA) and the trident acetabular shell (Stryker Ltd, Mahwah, NJ, USA). Patients in both treatment groups will undergo the same postoperative rehabilitation programme prior to discharge. All patients will receive postoperative patientcontrolled analgesia (PCA) with the background intravenous morphine infusion rate set at $0.5 \mathrm{mg} / \mathrm{h}$, bolus dose of $2 \mathrm{mg}$, and lockout period of $10 \mathrm{~min}$. Additional oral paracetamol and ibuprofen may be administered by the nursing staff if requested by the patient. The PCA will be stopped $24 \mathrm{~h}$ after surgery and converted to an oral regimen of regular paracetamol, ibuprofen, and dihydrocodeine, with oral morphine available for breakthrough pain.

\section{Outcomes}

All study patients will undergo review by two blinded observers (one orthopaedic registrar and one clinical research fellow) at 2 weeks, 6 weeks, 6 months, 1 year, and 2 years following surgery. During these follow-up times, predefined clinical, functional, and radiological outcomes will be recorded by these observers using case report forms (CRFs). The following outcomes will be recorded in all study patients:

1. Operating time (minutes)

2. Time to hospital discharge (hours)

3. Pain scores on the VAS and analgesia requirements preoperatively, during the inpatient admission, and postoperatively at 6 weeks, 6 months, 1 year, and 2 years

4. Patient satisfaction as assessed using the Musculoskeletal Outcomes Data Evaluation and Management System (MODEMS) preoperatively and postoperatively at 6 weeks, 6 months, 1 year, and 2 years following surgery [9]

5. Patient-reported outcome measures including Oxford hip score (OHS), Harris hip score (HSS), hip disability and osteoarthritis outcome score
(HOOS), University College Hospital hip (UCH) score, and Western Ontario and McMaster Universities Arthritis Index (WOMAC) preoperatively and postoperatively at 6 weeks, 6 months, 1 year, and 2 years following surgery

6. Health-related quality of life as measured using European Quality of Life questionnaire with 5 dimensions for adults (EQ-5D) preoperatively and postoperatively at 6 weeks, 6 months, 1 year, and 2 years

7. Accuracy of achieving the planned implant positioning and hip biomechanics as assessed using plain pelvic and hip radiographs preoperatively and postoperatively prior to discharge

8. Mobilisation distance (metres) and use of mobility aids preoperatively and postoperatively at 6 weeks, 6 months, 1 year, and 2 years

9. Range of movement (degrees) in hip joint preoperatively and postoperatively at 6 weeks, 6 months, 1 year, and 2 years

10. Muscle group strength testing in hip joint preoperatively and postoperatively at 6 weeks, 6 months, 1 year, and 2 years as measured using a dynamometer

11. Gait analysis performed postoperatively at 6 weeks, 6 months, 1 year, and 2 years as assessed using force plate treadmill testing

12. Femoral and acetabular implant early migration as assessed using RSA performed postoperatively at 2 weeks, 6 weeks, 6 months, 1 year, and 2 years

13. Resource use and cost-effectiveness including comparisons between the two treatment groups relating to operating time, theatre efficiency, equipment and sterilisation costs, analgesia requirements, inpatient rehabilitation, time to discharge, outpatient followup, functional outcomes, quality of life, return to work, additional imaging costs, and complications with their respective treatments

14. Complications

\section{Blinding}

All patients and clinical staff recording postoperative study outcomes will remain blinded to the treatment group. Study patients will be identifiable with a unique study number. Only the research physiotherapist will have the key to identify individual patients and their respective treatment arm. Any documents related to the study will be archived directly at the study site by the research physiotherapist within a locked filing cabinet in a locked research office. This office has swipe card access with onsite security and 24-h CCTV surveillance. Patient data will be logged electronically using each patient's unique identification number with computer software on an encrypted, password-protected research computer. 


\section{Sample size}

Prior to commencement of the study, a sample size of 80 patients ( 40 patients in each treatment arm) was selected to achieve a power of $80 \%(1-\beta)$ for assessing differences in pain scores on the visual analogue scale at $24 \mathrm{~h}$ after surgery between the two treatment groups, using an effect size of 0.67 , alpha value of 0.05 , and accounting for $10 \%$ sample size attrition rate during the 2year follow-up period [17, 20]. A sample size attrition rate of $10 \%$ was selected based on previous randomised controlled studies performed in our treatment centre.

\section{Statistical analysis}

The analysis of the per-protocol population will be considered the primary analysis. The differences between the PA and DSA groups will be analysed by calculating the difference from baseline, per patient, and a twosided confidence interval for the difference between the changes from baseline values will be calculated. This confidence interval will cover the true difference in the percentage change from baseline with a probability of 95\%. The following statistical methods will be employed to analyse the data: descriptive statistics, independent $t$ test, paired $t$ test, analysis of variance, Fisher exact test, chi-square test, and graphical displays. Assumptions of normality will be tested with the D'Agostino test. Assumptions of homogeneity of variance will be tested with Levene's test. If the distributional assumptions are (severely) violated, non-parametric techniques, such as Mann-Whitney's test will be employed. In the event that the DSA is converted to PA intraoperatively, analysis will be performed using the intention-to-treat population and the treatment actually received by the patients. Intraoperative conversion from DSA THA to PA THA will be documented and presented and published as part of the study. Statistical significance is set at a $p$ value < 0.05 for all analyses and all statistical analysis will be performed using SPSS software version 26 (SPSS Inc., Chicago, IL, USA).

\section{Adverse events}

Adverse events are defined as any untoward medical occurrence in a patient or study participant, which does not necessarily have a causal relationship with the procedure involved. A serious adverse event (SAE) is an adverse event that results in hospitalisation or prolongation of existing hospitalisation, persistent or significant disability or incapacity, life-threatening clinical sequelae, or death. All SAEs during the protocol treatment will be reported directly to the sponsor using the SAE web form. The chief investigator will also assess the SAE for severity, causality, seriousness, and expectedness using pre-existing criteria provided by the sponsor and inform the Data Safety Monitoring Board (DSMB) within 3 days of the initial observation of the event. The protocol treatment period is defined as the period from the day that the first study patient is recruited into the trial to the day that the final study patient has completed the 2year follow-up. The chief investigator will also inform the London-Fulham Research Ethics Committee and local Health Research Authority within 3 days of the SAE taking place. Safety aspects of the study are closely monitored by the sponsor and DSMB using unblinded data for its judgment. In cases where the SAE arises due to a problem with the study implants, Stryker Limited will also be notified within 2 days of the event taking place. The chief investigator will record the following: onset date, complete description of the event, severity, duration, action taken, and outcome for each SAE. The chief investigator will also provide regular updates of all SAEs to the London-Fulham Research Ethics Committee, local Health Research Authority, DSMB, and sponsor.

\section{Data management}

Onsite monitoring visits shall occur throughout the course of the clinical study by the chief investigator. The chief investigator shall permit and assist the sponsor (should they chose to monitor the study) to carry out verification of all study forms against data in the source documents, which shall occur as per the departmental policy for undertaking such activities. University College Hospital recognises that there is an obligation to archive study-related documents at the end of the study. The study master file will be archived at University College London in accordance with the University College Hospital Standard Operating Procedure for Archiving of Investigator Site File (ISF) and Pharmacy Site File (PSF). It will be archived for a minimum of 5 years from the study end, and no longer than 30 years from the study end.

\section{End of protocol treatment}

Reasons for going off study protocol include:

- Completion of the last follow-up visit 2 years after surgery

- Patient non-compliance or withdrawal (the reason for discontinuation will be recorded in the case report form)

- Intercurrent death

All patients included in this study are free to withdraw from the study at any time without compromise to their future treatment. On withdrawal, patients will revert to the standard follow-up regimen for routine (non-study) THA at the study site. The end of the study form will be completed and the reason for withdrawal documented. This form will also be completed if the patient is lost to 
follow-up or dies during the course of the study. Data to the point of discontinuation will be used for analysis.

\section{Monitoring}

The chief investigator will monitor the progress of the clinical study in the form of monthly research meetings for those involved in the trial. The chief investigator will be responsible for day-to-day monitoring and management of the study. The UCLH/UCL/Joint Research Office, on behalf of UCL as the sponsor, will monitor and conduct random audits on a selection of studies in its clinical research portfolio. Monitoring and auditing will be conducted in accordance with the Department of Health Research Governance Framework for Health \& Social Care (April 2005) and in accordance with the sponsor's monitoring and audit policies and procedures. As per the protocol, the principal investigator will email the sponsor twice yearly with the following: delegation log, adverse event log, deviation log, and any annual progress reports sent to the Ethics committee.

\section{Peer review}

The study protocol has undergone independent external peer reviewer. The suggestions and recommendations for improvement to the study design were implemented. The reviewers, sponsor, and London-Fulham Research Ethics Committee reviewed the revised protocol documents and confirmed that all queries and suggestions had been fully addressed.

\section{Discussion}

THA is an effective procedure for relieving pain, restoring function, and improving the quality of life in patients with end-stage hip osteoarthritis. The surgical approach in THA is important as it influences postoperative gait, hip stability, and muscle function $[2-5,7,16,20]$. The DSA is a minimally invasive modification of the PA that preserves the iliotibial band and short external rotators except for the piriformis or conjoint tendon during THA $[17,19,21]$. This prospective double-blinded randomised control trial will include 80 patients with symptomatic hip osteoarthritis undergoing primary THA. Following informed consent, patients will be randomised to undergo THA using the PA (control group) or DSA (investigation group) at a ratio of 1:1 using an online random number generator. Blinded observers will review patients at regular intervals for 2 years after surgery to record predefined study outcomes relating to postoperative rehabilitation, clinical progress, functional outcomes, accuracy of achieving planned implant positioning and hip biomechanics, cost-effectiveness, and complications. Gait analysis will be undertaken using force plate treadmills and implant stability assessed using RSA [22-24]. The findings of this study will provide an improved understanding of differences in the PA versus DSA for THA with respect to patient satisfaction, functional outcomes, implant position, implant survivorship, gait, costeffectiveness, and complications.

\section{Trial status}

Protocol: version 1.0; date 01 September 2017

Patient recruitment date: 1 June 2018

Estimated completion of recruitment date: 1 December 2020

Estimated completion of final follow-up: 1 December 2022

\section{Abbreviations}

CRF: Case report form; DSA: Direct superior approach; DSMB: Data Safety Monitoring Board; EQ-5D: European Quality of Life questionnaire with 5 dimensions for adults; HHS: Harris hip score; HOOS: Hip disability and osteoarthritis outcome score; OHS: Oxford hip score; PA THA: Posterior approach; SAE: Serious adverse event; SF-12: Short form health survey of 12 items; WOMAC: Western Ontario and McMaster Universities Arthritis Index

\section{Acknowledgements}

Nil

\section{Sponsor}

The study is sponsored by University College London, 235 Euston Road, Bloomsbury, London, NW1 2BU, UK (reference: 231646).

\section{Authors' contributions}

BK and JT performed background research, identified gaps in medical literature, created study objectives, trial design, created CRFs, attended Research Ethics Committee meeting, helped write study protocol, and prepared National Institute for Health Research (NIHR) Clinical Research Network (CRN) Industry Costing Template.

SK, JSC, and FSH helped write study protocol. The authors read and approved the final manuscript.

\section{Funding}

No funding was received for this study.

\section{Availability of data and materials}

The datasets used and/or analysed during the current study are available from the corresponding author on reasonable request.

\section{Ethics approval and consent to participate}

The study has been reviewed and approved for patient recruitment by the London-Fulham Research Ethics Committee, UK (reference: 18/LO/1972). Written informed consent will be obtained from participants during recruitment on site and prior to data collection. Consent to use the data collected for scientific reporting and publication will also be obtained at the same time as the consent to participate.

\section{Consent for publication}

The findings of this research will be published in peer-review journals. All study patients will provide informed consent for the publication of anonymised patient data and study findings. Authorship will reflect the amount of time spent designing the study, collating the data, analysing the results, and writing the manuscript.

\section{Competing interests}

FSH reports board membership of the Bone and Joint Journal and the Annals of the Royal College of Surgeons; consultancy for Smith \& Nephew, Corin, MatOrtho and Stryker; payment for lectures including service on speakers' bureaus for Smith \& Nephew and Stryker; and royalties paid by Smith \& Nephew, MatOrtho, Corin and Stryker, all outside the submitted work. SK reports consultancy, payment for lectures including service on speakers' bureaus, payment for development of education presentations, and travel/ 
accommodations/meeting expenses for Smith and Nephew and AO, all outside the submitted work.

All other authors declare no competing interests.

Received: 2 March 2020 Accepted: 6 June 2020

Published online: 19 June 2020

\section{References}

1. Amanatullah DF, Masini MA, Roger DJ, et al. Greater inadvertent muscle damage in direct anterior approach when compared with the direct superior approach for total hip arthroplasty. Bone Joint J. 2016;98-b(8):1036-42.

2. Barrack RL, Krempec JA, Clohisy JC, et al. Accuracy of acetabular component position in hip arthroplasty. J Bone Joint Surg Am. 2013;95(19):1760-8.

3. Barrack RL, Paprosky W, Butler RA, et al. Patients' perception of pain after total hip arthroplasty. J Arthroplast. 2000;15(5):590-6.

4. Berger RA. Total hip arthroplasty using the minimally invasive two-incision approach. Clin Orthop Relat Res. 2003;417:232-41.

5. Bergin PF, Doppelt JD, Kephart CJ, et al. Comparison of minimally invasive direct anterior versus posterior total hip arthroplasty based on inflammation and muscle damage markers. J Bone Joint Surg Am. 2011;93(15):1392-8.

6. Biedermann $\mathrm{R}$, Tonin $\mathrm{A}$, Krismer $\mathrm{M}$, et al. Reducing the risk of dislocation after total hip arthroplasty: the effect of orientation of the acetabular component. J Bone Joint Surg (Br). 2005;87(6):762-9.

7. Bremer AK, Kalberer F, Pfirrmann CW, et al. Soft-tissue changes in hip abductor muscles and tendons after total hip replacement: comparison between the direct anterior and the transgluteal approaches. J Bone Joint Surg (Br). 2011;93(7):886-9.

8. Fehring TK, Mason JB. Catastrophic complications of minimally invasive hip surgery. A series of three cases. J Bone Joint Surg Am. 2005;87(4):711-4.

9. Graham B, Green A, James M, et al. Measuring patient satisfaction in orthopaedic surgery. J Bone Joint Surg Am. 2015;97(1):80-4

10. Greidanus NV, Chihab S, Garbuz DS, et al. Outcomes of minimally invasive anterolateral THA are not superior to those of minimally invasive direct lateral and posterolateral THA. Clin Orthop Relat Res. 2013;471(2):463-71.

11. Hartzband MA. Posterolateral minimal incision for total hip replacement: technique and early results. Orthop Clin North Am. 2004;35(2):119-29.

12. Kayani B, Konan $\mathrm{S}$, Chandramohan $\mathrm{R}$, et al. The direct superior approach in total hip arthroplasty. Br J Hosp Med (Lond). 2019;80(6):320-4.

13. Kayani B, Konan S, Thakrar RR, et al. Assuring the long-term total joint arthroplasty: a triad of variables. Bone Joint J. 2019;101-b(1_Supple_A):11-8.

14. Lavernia C, D'Apuzzo M, Hernandez VH, et al. Patient-perceived outcomes in thigh pain after primary arthroplasty of the hip. Clin Orthop Relat Res. 2005; 441:268-73.

15. Lewinnek GE, Lewis JL, Tarr R, et al. Dislocations after total hip-replacement arthroplasties. J Bone Joint Surg Am. 1978;60(2):217-20.

16. Meermans $\mathrm{G}$, Konan S, Das R, et al. The direct anterior approach in total hip arthroplasty: a systematic review of the literature. Bone Joint J. 2017;99-b(6): 732-40.

17. Nam D, Meyer Z, Rames RD, et al. Is the direct superior, iliotibial bandsparing approach associated with decreased pain after total hip arthroplasty? J Arthroplast. 2017;32(2):453-7.

18. Pellicci PM, Bostrom M, Poss R. Posterior approach to total hip replacement using enhanced posterior soft tissue repair. Clin Orthop Relat Res. 1998;355: 224-8.

19. Penenberg BL, Bolling WS, Riley M. Percutaneously assisted total hip arthroplasty (PATH): a preliminary report. J Bone Joint Surg Am. 2008; 90(Suppl 4):209-20.

20. Poehling-Monaghan $\mathrm{KL}$, Kamath $\mathrm{AF}$, Taunton MJ, et al. Direct anterior versus miniposterior THA with the same advanced perioperative protocols: surprising early clinical results. Clin Orthop Relat Res. 2015;473(2):623-31.

21. Roger DJ, Hill D. Minimally invasive total hip arthroplasty using a transpiriformis approach: a preliminary report. Clin Orthop Relat Res. 2012; 470(8):2227-34

22. Ryd L, Albrektsson BE, Carlsson L, et al. Roentgen stereophotogrammetric analysis as a predictor of mechanical loosening of knee prostheses. J Bone Joint Surg (Br). 1995;77(3):377-83.

23. Selvik G. Roentgen stereophotogrammetry. A method for the study of the kinematics of the skeletal system. Acta Orthop Scand Suppl. 1989;232:1-51.

24. Selvik G, Alberius $P$, Aronson AS. A roentgen stereophotogrammetric system. Construction, calibration and technical accuracy. Acta Radiol Diagn (Stockh). 1983;24(4):343-52.
25. Timperley AJ, Biau D, Chew D, et al. Dislocation after total hip replacement there is no such thing as a safe zone for socket placement with the posterior approach. Hip Int. 2016;26(2):121-7.

\section{Publisher's Note}

Springer Nature remains neutral with regard to jurisdictional claims in published maps and institutional affiliations.
Ready to submit your research? Choose BMC and benefit from:

- fast, convenient online submission

- thorough peer review by experienced researchers in your field

- rapid publication on acceptance

- support for research data, including large and complex data types

- gold Open Access which fosters wider collaboration and increased citations

- maximum visibility for your research: over $100 \mathrm{M}$ website views per year

At $\mathrm{BMC}$, research is always in progress.

Learn more biomedcentral.com/submissions 